\title{
Wavefront sensors for adaptive optical systems
}

\author{
V.P. Lukin, N.N. Botygina, O.N. Emaleev, P.A. Konyaev \\ Zuev Institute of Atmospheric Optics, Russian Academy of Sciences, Siberian Branch, Academicheskii ave.1, 634021, \\ Tomsk, Russia, petrkonyaev@gmail.com
}

\begin{abstract}
A high precision Shack-Hartmann wavefront (WF) sensor has been developed on the basis of a low-aperture offaxis diffraction lens array. The device is capable of measuring WF slopes at array sub-apertures of $640 \times 640 \mu \mathrm{m}$ in size with an error not exceeding 4.80 arcsec (0.15 pixel), which corresponds to the standard deviation equal to $0.017 \lambda$ at the reconstructed WF with wavelength $\lambda$. Also the modification of this sensor for adaptive system of solar telescope using extended scenes as tracking objects, such as sunspot, pores, solar granulation and limb, is presented.

The software package developed for the proposed WF sensors includes three algorithms of local WF slope estimation (modified centroids, normalized cross-correlation and fast Fourier-demodulation), as well as three methods of WF reconstruction (modal Zernike polynomial expansion, deformable mirror response function expansion and phase unwrapping), that can be selected during operation with accordance to the application.
\end{abstract}

Keywords: Wavefront sensor, solar and adaptive optics, turbulent atmosphere

\section{INTRODUCTION}

$\mathrm{T}$ HE RESULTS of recent works related to techniques and algorithms for wavefront (WF) measurement using Shack-Hartmann sensors show their high efficiency in solving very different tasks of applied optics [1]-[5]. The Shack-Hartmann sensors sensitive to small WF aberrations are used for adaptive optical systems (AOS), compensating the wave distortions caused by atmospheric turbulence.

The goal of this paper was to develop a sensitive ShackHartmann sensor with high precision WF measurement capability based on modern technology of optical elements making and new efficient methods and computational algorithms of WF reconstruction.

In the first half of this paper we describe three algorithms of local WF slope estimation and three methods of WF reconstruction used in our software. In the second half we describe the design and test results of WF sensors, developed in the Laboratory of Coherent and Adaptive Optics at the Institute of Atmospheric Optics, Russian Academy of Sciences, Siberian Branch.

\section{ALGORITHMS FOR LOCAL WAVEFRONT SLOPE ESTIMATION}

The wavefront $w(x, y)$ can be determined from the gradient vector equation

$$
\frac{\partial w}{\partial x} \boldsymbol{i}+\frac{\partial w}{\partial x} \boldsymbol{j}=S_{X} \mathbf{i}+S_{Y} \boldsymbol{j}
$$

where $S_{X}, S_{Y}$. are the local WF slopes. In Shack-Hartmann sensors these local slopes are measured by means of processing a diffraction pattern, obtained from the lens raster (micro-lens matrix) at sensor sub-images. To estimate WF slopes, the measurement data of current shifts of Hartmann pattern focal spots with respect to their unperturbed positions are used:

$$
\begin{array}{ll}
S_{x}[m]=\frac{1}{f}(x[m]-x 0[m]) ; & m=1, \ldots, N_{S} ; \\
S_{y}[m]=\frac{1}{f}(y[m]-y 0[m]) ; & m=1, \ldots, N_{S} ;
\end{array}
$$

here $x[\mathrm{~m}], y[\mathrm{~m}], x 0[\mathrm{~m}], y 0[\mathrm{~m}]-$ are coordinates of current and reference focal spot positions (measured e.g. on a reference Hartmann pattern with a plane WF), $f$ is the microlens focal length, $m$ is the spot order number, $N_{S}$ is the number of sub-images used on the Hartmann pattern.

The shift vectors $S_{x}, S_{y}$ in $x$ and $y$ directions are computed in different ways, such as finding the coordinates of Hartmann pattern spot centroids, cross-correlation technique, and Fourier demodulation technique. The centroid technique is used in processing of the Hartmann pattern obtained from point objects; the centroid coordinates here are computed on the two-dimensional grid $\left[N_{i} \times N_{j}\right]$, corresponding to the sensor pixel matrix (CCD matrix), by the following equations:

$$
\begin{gathered}
x_{c}=\frac{\sum_{i=1}^{N_{i}} x_{i} I_{i}}{\sum_{i=1}^{N_{i}} \sum_{j=1}^{N_{j}} I_{i j}}, \quad y_{c}=\frac{\sum_{j=1}^{N_{j}} y_{j} I_{j}}{\sum_{i=1}^{N_{i}} \sum_{j=1}^{N_{j}} I_{i j}}, \\
I_{i}=\sum_{j=1}^{N_{j}} I_{i j}, I_{j}=\sum_{i=1}^{N_{i}} I_{i j},
\end{gathered}
$$

where $i$ and $j$ are the numbers of an element (pixel) in a row and column, respectively, $N_{i}$ and $N_{j}$ are the numbers of elements in a row and column of the matrix, $I_{i j}$ is the measured signal of the pixel with the coordinates $i, j$. To decrease the errors of coordinates of the centroids caused by sensor noises, the weight functions and the "tracking window" algorithm are used [6]. 
The cross-correlation frame processing is used for Hartmann patterns from extended objects obtained, e.g., in adaptive solar telescopes, and the coordinates of maxima of mutual correlation functions of sub-apertures of a current and reference Hartmann pattern are found instead of centroid coordinates (Fig.1).

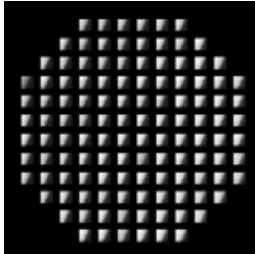

a

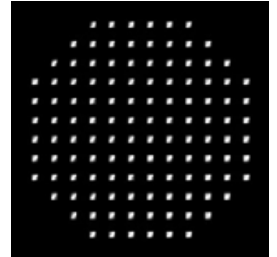

b

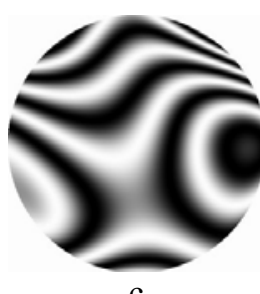

$\mathrm{c}$
Fig.1. Hartmann pattern (a) of the extended scene (sun disk limb), normalized cross-correlation function matrix (b), interferogram of reconstructed wavefront (c).

The normalized mutual correlation functions are computed either by algorithm of direct discrete convolution (for matrices $12 \times 12$ and smaller) or by algorithm of mixed radix fast Fourier transform [8] (for matrices larger than 12x12) by the following equations:

$$
\begin{aligned}
& C_{N}(i, j)=C(i, j) / C_{R}(i, j) \\
& C(i, j)=\sum_{l=0}^{N-1} \sum_{m=0}^{M-1} I(l, m) I_{R}(i+l, j+m) \\
& C_{R}(i, j)=\left[\sum_{l=0}^{N-1} \sum_{m=0}^{M-1} I^{2}(l, m) \sum_{l=0}^{N-1} \sum_{m=0}^{M-1} I_{R}^{2}(i+l, j+m)\right]^{1 / 2}
\end{aligned}
$$

where $I_{R}(l, m)$ is the intensity of reference frame and $I(l, m)$ is the intensity of current frame.

We have suggested and use the modified Fourier algorithm for computing the correlation function of low-contrast objects [6] in the software:

$$
\begin{aligned}
& C(i, j)=F^{-}\left\{F^{+}\{I(l, m)\} \cdot F^{+}\left\{I_{R}(l, m)\right\}\right. \\
& \left.\left.\cdot \exp \left(-a\left[\left(k_{x}-k_{x 0}\right)+\left(k_{y}-k_{y 0}\right)\right]\right)\right\}\right\},
\end{aligned}
$$

where $F^{+}, F^{-}$are the operators of direct and inverse FFT, respectively; the parameters of filtering function $a, k_{x 0}, k_{y 0}$ are fit experimentally in accordance with spatial frequencies of extended objects and noise.

To accelerate the computations, shifts of these maxima can be estimated by two-dimensional mutual intensity spectra at sub-images of current and reference frames [9]. After finding the integer coordinates $i_{m}, j_{m}$ of the maximum points of mutual correlation function, their values are adjusted using the quadratic approximation

$$
\begin{aligned}
& x_{m}=i_{m}+0.5 \cdot\left[C\left(i_{m}-1, j_{m}\right)-C\left(i_{m}+1, j_{m}\right)\right] / \\
& \left./\left[C\left(i_{m}-1, j_{m}\right)+C\left(i_{m}+1, j_{m}\right)-2 C\left(i_{m}, j_{m}\right)\right\}\right], \\
& y_{m}=j_{m}+0.5 \cdot\left[C\left(i_{m}, j_{m}-1\right)-C\left(i_{m}, j_{m}+1\right)\right] / \\
& \left./\left[C\left(i_{m}, j_{m}-1\right)+C\left(i_{m}, j_{m}+1\right)-2 C\left(i_{m}, j_{m}\right)\right\}\right] .
\end{aligned}
$$

Note that the cross-correlation algorithms, if applied to point objects, give the results exceeding and agreeing with those of the centroid method; hence, the former could be used as alternate methods due to their low sensitivity to sensor noises, e.g., CCD matrix.

Wavefront slopes in the Fourier demodulation method are measured without direct finding of Hartmann pattern spot shifts [10], [11]. This method is also of low sensitivity to sensor noises and has the advantage of speed for a large number of sub-apertures. The idea of the method is following.

In the case of WF aberrated by function $S(x, y)$, a Hartmann pattern could be represented in the form of regular two-dimensional lattice:

$$
\begin{aligned}
& I(x, y) \approx \cos \left[k_{x} \cdot x+f \cdot S_{x}(x, y)\right]+ \\
& +\cos \left[k_{y} \cdot y+f \cdot S_{y}(x, y)\right]
\end{aligned}
$$

where $k_{x}=2 \pi / p_{x}, k_{y}=2 \pi / p_{y}$ are the spatial frequencies of micro-lens raster with the periods $p_{x}, p_{y}$.

To find the local slopes $S_{x}, S_{y}$, the relation connecting Fourier transforms of Hartmann pattern and phase gradients is used:

$$
\begin{aligned}
& F\{I(x, y)\} \approx F\left\{\exp \left(-i \cdot f \cdot S_{x}(x, y)\right)\right\} \cdot \delta\left(v_{x}-k_{x}, v_{y}\right)+ \\
& +F\left\{\exp \left(i \cdot f \cdot S_{x}(x, y)\right)\right\} \cdot \delta\left(v_{x}+k_{x}, v_{y}\right) \\
& +F\left\{\exp \left(-i \cdot f \cdot S_{y}(x, y)\right)\right\} \cdot \delta\left(v_{x}, v_{y}-k_{y}\right) \\
& +F\left\{\exp \left(i \cdot f \cdot S_{y}(x, y)\right)\right\} \cdot \delta\left(v_{x}, v_{y}+k_{y}\right)
\end{aligned}
$$

here $v_{x}, v_{y}$ are the spatial frequencies, $F\{\}$ is the Fourier transform operator. The side lobes of high orders $(m, n=1,2, \ldots)$ of two-dimensional Fourier spectrum of the Hartmann pattern are well separated in the coordinates of spatial frequencies, if the number of micro-lenses is sufficiently large and the aberration spectrum is localized.

Phase gradients are defined after low-frequency filtration separately in each direction according to the algorithm:

$$
\begin{aligned}
& S_{x}(x, y) \approx \arg \left\{F^{-}\left\{F^{+}\{I(x, y)\} \cdot \Phi_{x}\left(v_{x}, v_{y}\right)\right\},\right. \\
& S_{y}(x, y) \approx \arg \left\{F^{-}\left\{F^{+}\{I(x, y)\} \cdot \Phi_{y}\left(v_{x}, v_{y}\right)\right\},\right.
\end{aligned}
$$

where $\Phi_{x}\left(v_{x}, v_{y}\right), \Phi_{y}\left(v_{x}, v_{y}\right)$ are the filtering functions for the gradients over the coordinates $x$ and $y$, respectively:

$$
\begin{aligned}
& \Phi_{x}\left(v_{x}, v_{y}\right)=\Phi_{y}\left(v_{x}, v_{y}\right)=1, \\
& \text { if }\left(v_{x}-k_{x}\right)^{2}+\left(v_{y}-k_{y}\right)^{2} \leq\left(k_{0} / 2\right)^{2}, \\
& \Phi_{x}\left(v_{x}, v_{y}\right)=\Phi_{y}\left(v_{x}, v_{y}\right)=0, \\
& \text { if }\left(v_{x}-k_{x}\right)^{2}+\left(v_{y}-k_{y}\right)^{2}>\left(k_{0} / 2\right)^{2},
\end{aligned}
$$


here $k_{0}=2 \pi / p$ is the carrier spatial frequency of the Hartmann pattern with the period $p$.

\section{ALGORITHMS FOR WAVEFRONT RECONSTRUCTION FROM LOCAL SLOPES}

In the modal reconstruction method, the calculated WF is represented as the series expansion in terms of the basis function $\left\{Z_{k}\right\}$, defined on the whole sensor aperture:

$$
w(x, y)=\sum_{k=1}^{N_{Z}} c_{k} Z_{k}(x, y)
$$

where $N_{Z}$ is the number of basis functions. The parameters $N_{\mathbf{Z}}$ and $N_{\boldsymbol{S}}(2)$ determine the accuracy of WF representation, on the one hand, and influence the computation speed in the reconstruction algorithm, on the other hand. To find the expansion coefficient $c_{k}$, the rms error minimization procedure is used:

$$
\begin{aligned}
& \varepsilon^{2}=\sum_{m=1}^{N_{S}}\left[S_{x}[m]-\sum_{k=1}^{N_{Z}} c_{k} \frac{\partial}{\partial x} Z_{k}(x, y)\right]^{2}+ \\
& +\sum_{m=1}^{N_{S}}\left[S_{y}[m]-\sum_{k=1}^{N_{Z}} c_{k} \frac{\partial}{\partial y} Z_{k}(x, y)\right]^{2},
\end{aligned}
$$

which results in the matrix equation $\boldsymbol{S}=A \boldsymbol{c}$, having the solution

$$
\boldsymbol{c}=B \boldsymbol{S}, B=\left(A^{T} A\right)^{-1} A^{T}
$$

The reconstruction matrix $B$ is calculated when specifying the sensor geometry, which essentially accelerates the realtime computations.

In applications connected with measurements of WF aberrations, such as applied optics, atmospheric and adaptive optics, etc., the basis of Zernike polynomials orthogonal within the circle of the unit radius $\rho$ is usually chosen as basic function (8):

$$
\begin{aligned}
& Z_{k}(\rho, \theta)=N_{n}^{m} R_{n}^{|m|}(\rho) \cos (m \theta), \\
& m \geq 0,0 \leq \rho \leq 1,0 \leq \theta \leq 2 \pi, \\
& Z_{k}(\rho, \theta)=-N_{n}^{m} R_{n}^{|m|}(\rho) \sin (m \theta), \\
& m<0,0 \leq \rho \leq 1,0 \leq \theta \leq 2 \pi,
\end{aligned}
$$

using the continuous numbering of polynomials and OSA standard normalization according to the equations

$k=(n(n+2)+m) / 2$

$N_{n}^{m}=\sqrt{2(n+1) /\left(1+\delta_{m 0}\right)}$,

$\delta_{M 0}=1, m=0 ; \delta_{M 0}=0, m \neq 0$,

$R_{n}^{|m|}(\rho)=\sum_{l=0}^{(n-|m|) / 2}(-1)^{l}(n-l) ![l !((n+|m|) / 2-l) !((n-|m|) / 2-l) !]^{-1} \rho^{n-2 l}$
To calculate the Zernike polynomials and their derivatives, Horner's effective recurrent algorithms are used. If the purpose of WF reconstruction is control of a deformable mirror, then the experimentally measured mirror response functions are to be chosen as the basis.

Phase reconstruction by the Fourier demodulation method requires eliminating discontinuity of the phase surface calculated with respect to modulo $2 \pi$. The phase unwrapping algorithm based on two-dimensional FFT was suggested in [12] and developed in [13]. The unwrapping procedure consists in filtration of the Fourier transform of the phase gradient integral along $x$ and $y$ directions. The local slopes $S_{x}[i, j], S_{y}[i, j]$, specified on the grid $\left[N_{i} \times N_{j}\right]$, are added with the periodic boundary conditions:

$$
\begin{aligned}
& S_{x}[-1, j]=-S_{x}[0, j], \\
& S_{x}\left[N_{i}, j\right]=-S_{x}\left[N_{i}-1, j\right], \\
& S_{y}[i,-1]=-S_{y}[i, 0], \\
& S_{y}\left[i, N_{j}\right]=-S_{y}\left[i, N_{j}-1\right] .
\end{aligned}
$$

The algorithm of mixed radix fast 2-D Fourier transform is used for reconstruction of the unwrapped continuous surface $\varphi[i, j]$ from the wrapped phase according to the expression

$$
\begin{aligned}
& F\{\varphi[i, j]\}=F\{\rho[i, j]\}) / 2\left\{\cos \left(\pi l / N_{i}\right)+\right. \\
& \left.+\cos \left(\pi m / N_{j}\right)-2\right\}
\end{aligned}
$$

where $F$ is the discrete Fourier transform operator, and the function

$$
\begin{aligned}
& \rho[i, j]=S_{x}[i, j]-S_{x}[i-1, j]+ \\
& +S_{y}[i, j]-S_{y}[i, j-1]
\end{aligned}
$$

is the sum of gradient differences of the wrapped phase along $x$ and $y$ directions, respectively.

\section{WAVEFRONT SENSOR DESIGN AND RESULTS}

Shack-Hartmann sensors sensitive to small WF aberrations are used for adaptive optical systems (AOS) compensating the aberrations caused by atmospheric turbulence. An increase in the sensor sensitivity is attained by means of an increase in the focal length of micro-lens raster and a decrease in the pixel size of the sensor. But an increase in video camera resolution results in undesirable AOS frequency band reduction, since the Hartmann pattern processing time increases, and producing of raster of lowaperture refractive micro-lenses with full filling and high repeatability of micro-lens parameters presents a serious task. The diffraction raster manufacturing technology allows solving this difficulty.

We have used the diffractive raster [14] for WF sensor design. The raster consists of tightly packed square lowaperture diffraction elements of 640x640 $\mu \mathrm{m}$ in size. Each element is a small off-axis fragment of a large long-focus diffraction lens. The numerical aperture (NA) of a separate element is 0.005 . 
A digital video camera DALSA CAD6-512 (Canada) is used as a detector in the WF sensor; its resolution is $532 \times 516$ pixels $(1$ pixel $=10 \mu \mathrm{m})$, frame frequency is 262 frames per second. The video camera is connected with a computer through a Coreco PC-DIG frame grabber (Canada).

The laboratory-bench test of the WF sensor [7] has shown that the rms deviation of the measured local slopes does not exceed 4. 80 arcseconds. The rms deviation of centroid shifts of the diffraction images in the focal plane does not exceed 0.15 pixels.

The diffractive raster with $8 \times 8$ elements, laser beam with plane wavefront and the reference optical wedge were used in this experiment. Fig.2a shows the superposed trajectories of centroid shifts of 64 diffraction images when rotating the optical wedge round the optical axis through a full $360^{\circ}$. The mean centroid shifts of all the above images are shown in Fig.2b and Fig.2c at each fixed wedge position. Each following position differs from the previous one by rotation of the optical wedge through $30^{\circ}$ round the system optical axis. The vertical segments show the rms shift deviations.

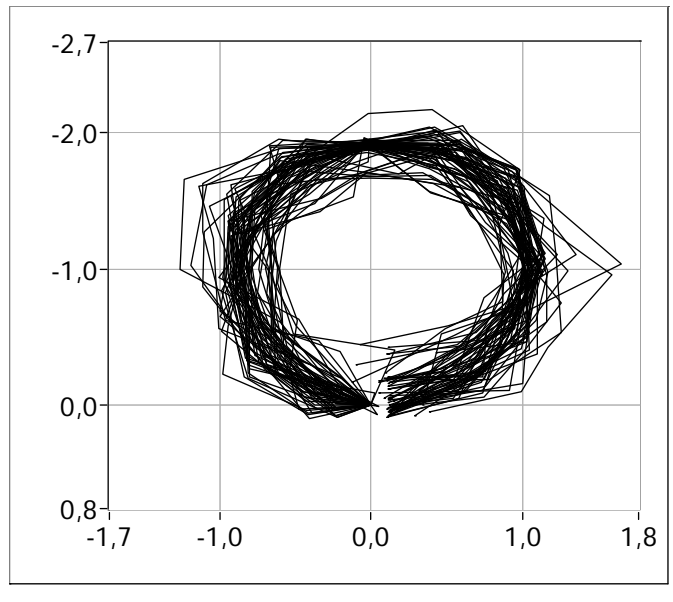

a

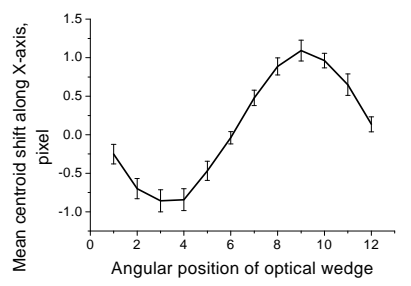

b

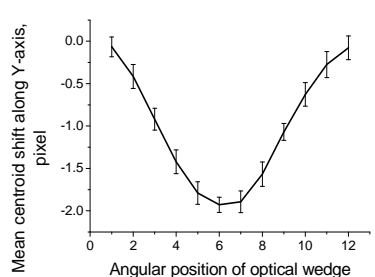

c
Fig.2. Shifts of the centroids of diffraction images at optical wedge rotation

Noncoincidence of the trajectories is connected with the errors in manufacturing of optical elements, WF aberrations of laser radiation, and noises of recording system.

If the integral measurement error is 0.15 pixels, then the rms deviation from flatness of reconstructed WF does not exceed $0.017 \lambda$ ( $\lambda=0.63 \mu \mathrm{m}$ is the wavelength of incident radiation) [7].

The use of low-aperture off-axis diffraction lenses with high quality of WF formation and high repeatability of the parameters of separate elements in the sensor allowed us to record local WF slopes with a high angular resolution and to measure aberrations caused by atmospheric turbulence.

Fig. 3 shows the sensor's test-bed view along with the measurement results of WF aberrations of a collimated laser beam of $100 \mathrm{~mm}$ in diameter with a radiation wavelength of $0.63 \mu \mathrm{m}$, propagating along a horizontal atmospheric path of $100 \mathrm{~m}$ in length. Table 1 gives the coefficients of Zernike polynomials in $\lambda$ fractions.

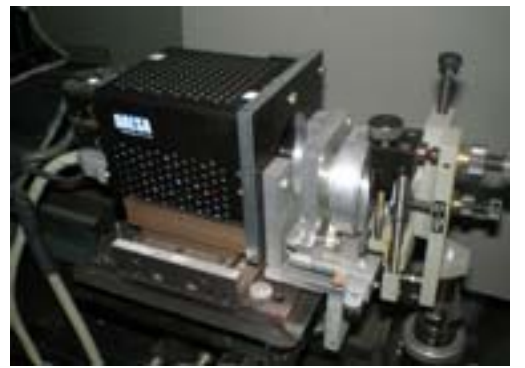

a

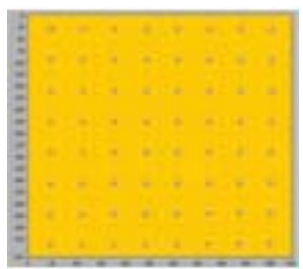

b

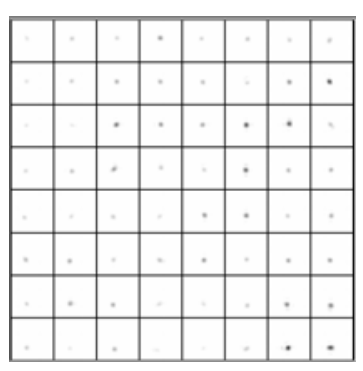

c

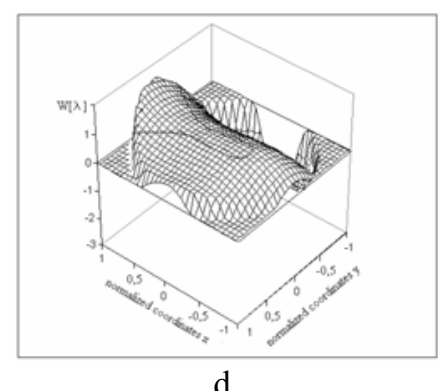

d

Fig.3. Sensor test-bed view (a) and measurement results: Hartmann pattern at the beginning (b) and at the end (c) of the path, wave aberration function(d) at the receiver input aperture ( $73 \mathrm{~mm}$ in diameter)

$$
\begin{aligned}
& \mathrm{C}_{1}=-0.552807 \\
& \mathrm{C}_{2}=1.08023 \\
& \mathrm{C}_{3}=-0.0145726 \\
& \mathrm{C}_{4}=-0.436019 \\
& \mathrm{C}_{5}=0.607858 \\
& \mathrm{C}_{6}=-0.262549 \\
& \mathrm{C}_{7}=0.116933 \\
& \mathrm{C}_{8}=-0.230521 \\
& \mathrm{C}_{9}=-0.152966 \\
& \mathrm{C}_{10}=-0.185922 \\
& \mathrm{C}_{11}=0.0234221 \\
& \mathrm{C}_{12}=0.0760849 \\
& \mathrm{C}_{13}=-0.171276 \\
& \mathrm{C}_{14}=0.1081888
\end{aligned}
$$

Table1

tilt in $\mathrm{x}$-direction tilt in y-direction astigmatism defocus astigmatism trefoil coma coma trefoil quatrefoil secondary astigmatism spherical aberration secondary stigmatism quatrefoil 
The Shack-Hartmann sensor has been developed on the base of a matrix of low-aperture off-axis diffraction microlenses for measurements of atmospheric turbulence parameters on a laser radiation propagation path and as an element of adaptive image-forming optical system.

Adaptive systems of solar telescopes use extended scenes, such as sunspots, pores and solar granulation, as tracking objects. In a Shack-Hartmann sensor of solar adaptive systems, each raster element forms the image of a chosen fragment of the solar disk. A sensor-recording Hartmann pattern is an image array of this fragment. The incident WF error results in shift of each image. The incident WF is reconstructed on the base of the measured shifts.

The Shack-Hartmann sensor has been developed for the AOS of Big Solar Vacuum Telescope (BSVT) of the Baikal Astrophysical Observatory, consists of the raster of square diffraction micro-lenses with the numerical aperture 0.019 and a GE-680 "Prosilica" video camera (Canada) with a resolution of $640 \times 480$ pixels $(1$ pixel $=7.4 \mu \mathrm{m})$. The WF sensor operates with broadband (10nm FWHM) visible light at about $535 \mathrm{~nm}$. A field of view of the BSVT adaptive system does not exceed 40 arcseconds.

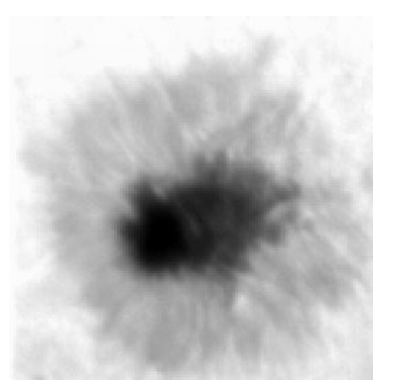

a

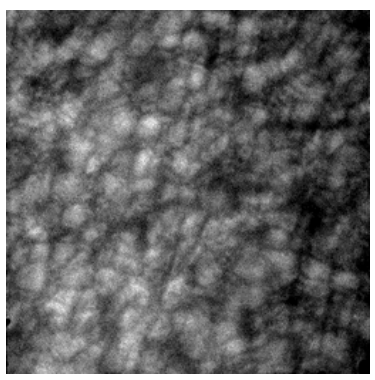

c

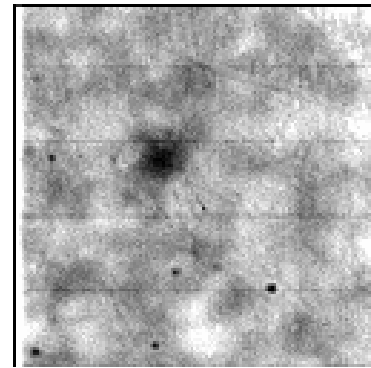

b

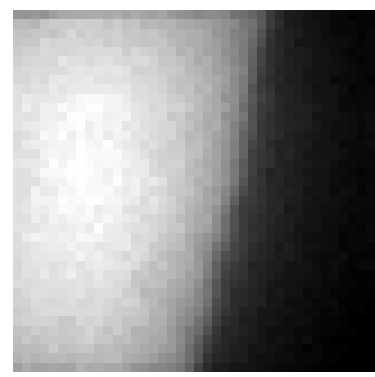

d
Fig.4. Extended scenes on the sun surface (Big Solar Vacuum Telescope on the Baikal Astrophysical Observatory): sunspot (a), pore (b), "lucky snap" of granulation (c), sun limb (d)

Fig. 4 shows the images of solar disk fragments used by the adaptive system of BSVT as tracking objects. The choice of optimal technique of local slope measurements is provided for AOS operation with various fragments of the solar disk.

A solar granulation fragment is a preferred tracking object as granulation is always present on the solar disk. But the image of this object is of low contrast due to the physical nature of solar granulation. In addition, atmospheric turbulence and instrumental errors of solar telescopes essentially decrease the contrast of granulation image. Image transfer to the second focus in real telescopes with AOS with the use of additional optical elements also results in a contrast decrease. The contrast of BSVT-recording granulation image is within the $1-4 \%$ range depending on seeing conditions. Designing the AOS to be effectively operating by a solar granulation fragment is a complicated problem mainly due to the difficulty in measuring shifts of low-contrast images.

To operate with an image of solar granulation fragment, we have developed modified correlation algorithm [3], [6] for shift measurements. If the parameters of filtering function are chosen accurately, low frequencies are rejected in the spatial spectrum of illumination distribution, which results in background illumination flat-field over the sensor field of view, as well as high frequencies connected with faults of image recording. As a result, frequencies corresponding to grain sizes prevail in the transformed spatial spectrum, and the maximum connected with a solar granulation image shift becomes global in the mutual correlation function. In good seeing conditions, the developed algorithm provides WF sensor operations by a solar granulation fragment.

When the sensor operates by the solar limb, spot or its segment, normalized mutual correlation function (4) is used. To determine a shift of image fragment with a pore, both modified correlation algorithm (at low contrast of pore image) and algorithm with normalized correlation function can be used.

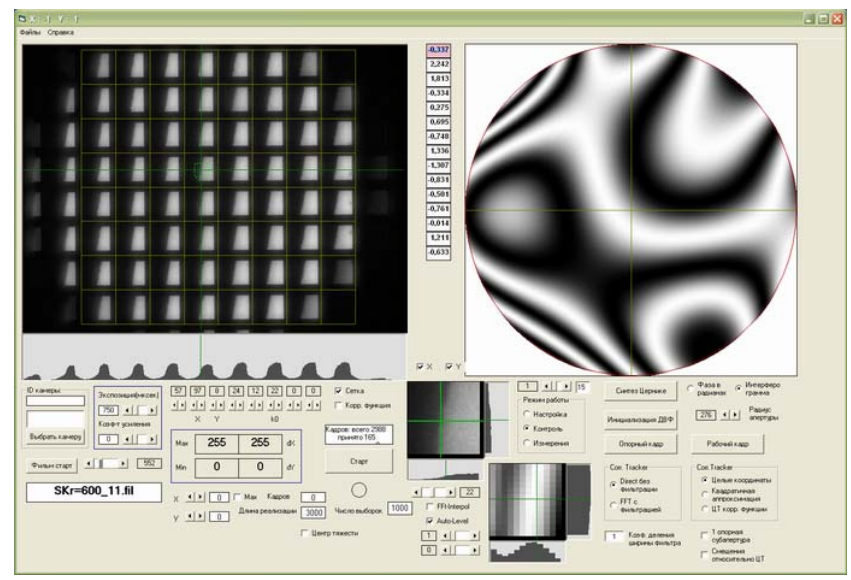

Fig.5. A screenshot of the Shack-Hartmann sensor program

The computational algorithms allowing WF reconstruction from the measurements of Shack-Hartmann patterns with high precision and the use of modern methods of analysis have been developed and realized in the form of an application package for Intel-based workstations in Windows environment.

Fig. 5 shows the main screen form of the Shack-Hartmann sensor program with controls and windows for imaging a current frame and measurement results. The program allows not only operating with "alive" images but also high speed processing (200 frames/sec) filmed Hartmann patterns. 


\section{CONCLUSION}

A high precision sensitive Shack-Hartmann sensor for atmospheric applications has been developed and tested using a laser as a light source. A universal design of this kind of device enables it to be used in different areas of adaptive optics (e.g., in solar and star astronomic telescopes).

The software developed for the proposed WF sensor has an algorithm selection capability of Hartmann pattern processing and WF reconstruction methods, which increases its range of application.

This work was performed within the framework of the integration project No.80 of the Siberian branch of the Russian Academy of Sciences "Development methods of precision astro-climate observations for ensuring adaptive optical system operation" and the program No.16 of the Presidium of the Russian Academy of Sciences, part 3 "Development of adaptive systems and atmospheric turbulence test set-ups for improving the solar astronomical observations".

\section{REFERENCES}

[1] Platt, B.C., Shack, R. (2001). History and principles of Shack-Hartmannn wavefront sensing. Journal of Refractive Surgery, 17 (9-10), S573-S577.

[2] Rimmele, T.R. (2004). Recent advances in solar adaptive optics. In Proceedings of the SPIE. Vol. 5490, 34-46.

[3] Lukin, V.P., Antoshkin, L.V., Botygina, N.N., Emaleev, O.N., Konyaev, P.A. (2006). Adaptive optical system for solar telescope. Optical Magazine, 73 (3), 55- 60.

[4] Canovas, C., Ribak, E.N. (2007). Comparison of Hartmannn analysis methods. Applied Optics, 46 (10), 1830-1835.

[5] Woger, F., Rimmele, T.R. (2009). Effect of anisoplanatism on the measurement accuracy of an extended source Hartmannn-Shack wavefront sensor. Applied Optics, 48 (1), A35-A46.
[6] Lukin, V.P., Antoshkin, L.V., Botygina, N.N., Emaleev, O.N., Grigor'ev, V.M., Konyaev, P.A., Kovadlo, P.G., Skomorovski, V.I., Yankov, A.P. (2006). Adaptive system of tip-tilt correction of image with modified correlation tracker for BSVT. Instrumentation, Mesure, Métrologie, 6 (1-4), 219223.

[7] Lukin, V.P., Botygina, N.N., Emaleev, O.N., Korolkov, V.P., Lavrinova, L.N., Nasyrov, R.K., Poleshchuk, A.G., Cherkashin V.V. (2009). ShackHartmannn sensor based on a low-aperture off-axis diffraction lens array. Avtometriya, 45 (2), 88-98.

[8] Singleton, R.C. (1969). An algorithm for computing the mixed radix fast Fourier transform. IEEE Transactions on Audio and Electroacoustics, AU-17 (N2), 93-103.

[9] Knutsson, P.A., Owner-Petersen, M. (2005). Extended object wave front sensing based on the correlation spectrum phase. Optics Express, 13 (23), 9527-9536.

[10] Pioneer, L.A., Gavel, D.T., Brase, J.M. (2002). Fast wave-front reconstruction in large adaptive optics systems with use of the Fourier transform. Journal of the Optical Society of America A, 19 (10), 2100-2111.

[11] Carmon, Y., Ribak, E.N. (2003). Phase retrieval by demodulation of a Hartmann-Shack sensor. Optics Communications, 215, 285-288.

[12] Freischlad, K.R., Koliopoulus, C.L. (1986). Modal estimation of a wave front from difference measurements using the discrete Fourier transform. Journal of the Optical Society of America A, 3 (11), 1852-1861.

[13] Pritt, M.D., Shipman, J.S. (1994). Least-squares twodimensional phase unwrapping using FFT's. IEEE Transactions on Geoscience and Remote Sensing, 32 (3), 706-708.

[14] Korolkov,V.P., Poleshchuk, A.G., Nasyrov, R.K. et al. (2007). Application of off-axis microlens array in low aperture Shack-Hartmannn sensor. In EOS Topical Meeting on Diffractive Optics, 20-23 November 2007. Barcelona, 240-241. 